\title{
HOW DOES ONE DETECT AN ACCRETION DISK?
}

\author{
Ronald H. Kaitchuck \\ Department of Physics and Astronomy \\ Ball State University \\ Muncie, IN 47306 \\ USA
}

\begin{abstract}
The history of the first detection of an accretion disk in a interacting binary system is presented. The nature of transient and permanent disks in Algol-type binaries is discussed as are the problems associated with the interpretation of emission line profiles from the disks in cataclysmic variable stars. The new technique of eclipse mapping from continuum photometry is reviewed. Some results of Doppler tomography of emission lines are presented.
\end{abstract}

\section{Introduction}

The first detection of an accretion disk was accidental and reported in a footnote in an observatory report. It was several years before its significance was understood. In 1934, Wyse (1934) was conducting a program to obtain spectra of the secondary stars in Algol-type binaries by observing during primary eclipse. For one such system, RW Tau, a spectrum obtained near the end of totality showed hydrogen emission lines on either side of the stellar absorption lines. These emission lines were very difficult to see outside of totality because of the brilliance of the uneclipsed primary star. It occurred to Wyse that the eclipse could be used to map the structure and location of the emission line region. During one eclipse, he obtained nine closely spaced spectra during the 80-minute totality. Unfortunately, even though he was using the Lick Observatory $3 \mathrm{~m}$ telescope, all his photographic plates were badly underexposed.

Seven years later A. Joy (1942) began a study of RW Tau. Although he suffered from the same instrumental limitations as Wyse (he could obtain no more than 2 to 3 spectra during totality) he sampled enough eclipses so that a pattern became apparent. Near the beginning of totality the emission lines were redshifted by $350 \mathrm{~km} \mathrm{~s}^{-1}$. At mid-eclipse they disappeared briefly and re-appeared near the end of totality with a blueshift of $350 \mathrm{~km} \mathrm{~s}^{-1}$. Joy offered a simple, but ingenious model to explain the observations. He proposed that the primary star was surrounded by a rotating gaseous ring. The observed emission line behavior could be explained as an eclipse of this ring by the secondary star. This model caught the imagination of many people. It even appeared as an illustration in one of the astronomy text books of that time (Skilling and Richardson 1947). No doubt, the picture that most people had in their minds was that of a gaseous version of the thin, Keplerian ring of Saturn. 
In the next few years, Struve and collaborators (1948, 1949; Struve and Huang 1957) discovered many more binary systems with gaseous rings. Rings were found to be fairly common in Algol-type binaries. In systems with orbital periods less than about 5 days (like RW Tau) the emission is weak and only visible near the bottom of eclipse. For systems with periods greater than about 5 days the emission is strong enough to be detectable outside of eclipse. Struve reasoned that in the longer period systems there is more room between the stars, and the rings are therefore larger and consequently brighter. Also, as Joy had noted earlier in RW Tau, the emission lines in the short-period Algols are highly variable with asymmetries in the emission strength of the red and blue-shifted sides of the ring.

In the years that followed the concept of mass transfer between the component stars of a binary system was introduced (Crawford 1955). Mass transfer was offered as the solution to the "Algol Paradox," where the less massive star is the more evolved. With this our modern ideas about stellar evolution in binary stars were born. The gaseous rings became recognized as a result of mass transfer and the accretion process, so they became known as accretion disks. The theory of accretion disk structure is now quite extensive. The "standard" picture is that of a thin, axisymmetric disk rotating in a very nearly Keplerian fashion. Viscosity in the gas causes it to radiate and lose kinetic energy. The process also transfers the angular momentum of the infalling gas outward where it is given to the orbital momentum via tidal interactions of the outer disk with the secondary star (Frank, King and Raine 1985). The disk provides a way to slowly lower gas down the potential well of the mass-gaining star and liberate potential energy.

\section{Types of Accretion Disks}

Binary stars span a vast range in their parameters such as orbital period, masses and mass transfer rates. Consequently, we shouldn't expect the accretion disks in these systems to look the same. The disks found in short-period Algol systems like RW Tau are rather small (see below) and with rather low accretion rates. As a consequence, the disks appear to be optically thin in the continuum but often optically thick in the emission lines (as judged from the Balmer decrement). In cataclysmic binaries, the disks are even smaller with higher accretion rates. In this case, a large portion is optically thick in the continuum and the disk is a dominant source of continuum light.

Even the kinematics of the disks differ. It was pointed out by Budding (1980) and Peters and Polidan (1984) that a useful diagram is a plot of the relative radius of the mass-gaining star $\left(R_{1} / a\right)$ versus the mass ratio $(q)$ of the binary. A survey of Algol systems in eclipse for disk emission (Kaitchuck, Honeycutt and Schlegel 1985) revealed a segregation in this diagram based on the character of disk emission (see Figure 1). Systems which failed to show emission plotted high in the diagram (i.e. a large relative size of the mass-gaining star), while systems which show strong permanent emission (the long-period Algols) plot low. Those systems with variable and transient emission (like RW Tau) fall in between. A curve can be superposed on this diagram which indicates relative distance of closest approach of a stream to the massgaining star as a function of mass ratio (Lubow and Shu 1975). The stars with permanent disk 
emission generally fall below this line (i.e. the stream misses the star). The systems with variable emission lie a short way above this line, indicating a collision of the stream near the trailing limb of the star. This strongly implies that we should expect a very different disk structure for systems which lie above and below this line. For the short-period Algol systems we perhaps are seeing the impact debris of a star/stream collision, for those below the line (e.g. the long-period Algols and cataclysmic variables) the stream will sweep around the star and form a normal disk which is stable and axisymmetric. For those systems which plot high in the diagram no disk forms, perhaps because the stream cleanly burrows into the star. Evidence for the penetration of the stream into the primary of $U$ Cep has been reported by Olson (1980).

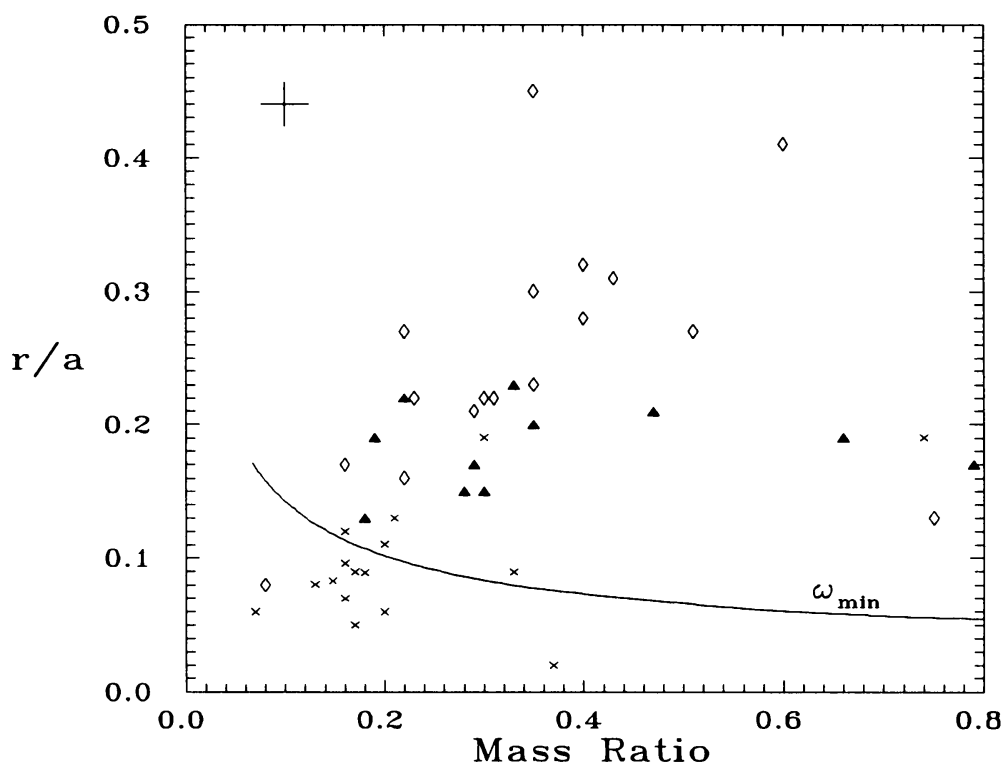

Figure 1. A plot of the relative size of the mass-gaining star versus mass ratio. The diamonds refer to systems without disks, the triangles to transient disk systems and the $\mathrm{X}$ 's to systems with permanent disks. The cross in the upper left represents a typical error bar. The curve marks the minimum distance between the accreting star and the stream trajectory.

\section{Transient Disks in Short-Period Algol Binaries}

It wasn't until 1978 that technology made possible Wyse's experiment of the mapping of the disk of RW Tau by time-resolved spectroscopy in eclipse (Kaitchuck and Honeycutt 1982). Studies using television-type detectors revealed much detail about the disk in RW Tau and other short-period Algol systems (Kaitchuck and Honeycutt 1982). For RW Tau it was possible to obtain 30 spectra during the 80 -minute totality. The results largely confirm the expectations for the unusual nature of this disk. Analysis of the changing Doppler components showed the disk to be smaller than previously estimated (on the assumption of circular Keplerian motion), with radii of only 1.1 to 1.7 times that of the central star. The leading and 
trailing sides of the disk are very asymmetric, both in terms of the line strengths and the radial velocities. The disk is extremely variable on a time scale of one orbital period. It sometimes disappeared for months at a time. For this reason it was labeled a transient disk. The observed radial velocities were significantly smaller than expected for gas moving in circular orbits at the measured radii. Furthermore, the line widths were usually at least a factor of two greater than that expected for a line broaden by rotation of a Keplerian disk. In at least two cases the velocity field on the leading side of the disk appeared reversed, i.e. the highest velocities occurred at the outer edge. Similar results have been obtained for U Cep (Kaitchuck, Honeycutt and Faulkner, 1989), TZ Eri (Kaitchuck and Park 1988) and SW Cyg (Kaitchuck 1989). This has lead to the conclusion that the disks in these binaries had supersonic turbulence. The same conclusion was independently reached for $\mathrm{U}$ Cep by Crawford(1981). Strong support for this picture came from IUE observations of emission lines in RW Tau (Plavec and Dobias 1983) and absorption lines in other Algol-like binaries (Peters and Polidan 1984). These observations showed high excitation lines characteristic of plasma with an electron temperature of $10^{5} \mathrm{~K}$. The latter authors coined the term the hot turbulent accretion region (HTAR) to describe this accretion flow.

But the picture may be more complicated. From time-to-time there have been brief reports in the literature of emission lines seen at the quadrature phases of short-period algols. To see this emission requires observations at $\mathrm{H} \alpha$ (because of the strong Balmer decrement) with a high signal-to-noise ratio. Most recently, such observations have been reported for Algol itself (Richards 1991; Honeycutt et al. 1991) and RW Tau (Honeycutt et al. 1991). These authors come to the same basic conclusion: there is another emission line producing region near the surface of the mass gaining star, but located along the line of stellar centers, i.e. not at the star/stream impact site. Doppler shifts of this emission indicate a velocity with the same direction and approximately the same magnitude as the disk. However, this region appears to be distinct from the disk. On one occasion, quadrature emission in RW Tau was observed, but eclipse observations made on the same orbit failed to detect any disk. Currently, it is very unclear as to why this separate emission region should exist. Perhaps this represents a pile up of material that managed to circumnavigate the star without being accreted and has been stopped by the "backside" of the stream.

\section{Permanent Disks in Long-Period Algols}

We expect that the long-period Algols will have disks closer to our expectations given the fact that the stream does not strike the mass-gaining star. In many ways the study of these systems is more difficult than it is for the short-period Algols. Due to their long orbital periods it is difficult or impossible to follow a complete eclipse in the course of a single night. Partial coverage of an eclipse doesn't map the disk very well. Using data from several different eclipses is subject to misinterpretation if the disk has changed between the various epochs of observation. Consequently there have been few attempts to study the emission line behavior through eclipse. In one case, $\mathrm{RY}$ Gem $(\mathrm{P}=9.3$ days), the total phase of eclipse lasts 4.5 hours and, with luck, it is possible to observe this in a single night. The results of such observations (Kaitchuck 1988) seem to show many of the same effects that are seen in the short-period Algols. RY Gem lies slightly below the curve in Figure 1, and it may be that a portion of the 
stream still grazes the limb of the mass-gaining star producing many of the characteristics of a transient disk.

The most extensive survey of the disk structure in long-period Algols is that of Peters (1989). She finds that for systems with periods greater than 6 days the disks appear to be stable with little variability in their emission lines. These disks are large, filling most of the Roche lobe of the mass-gaining star. The trailing side of the disk is usually brighter than the leading side, while the leading side is often more extended. Presumably, both these effects are due to the impact of the stream on the disk. That is, the stream makes the trailing side of the disk denser and brighter and disturbs the flow in the outer periphery producing a non-circular disk. While these systems show some differences from the standard disk model they may come much closer to this picture than any other type of disk.

\section{Disks in Cataclysmic Variables}

Given the early experiences with the Algol-type binaries, it is easy to see why the presence of double-peaked emission lines in cataclysmic variables (CVs) was quickly determined to be due to accretion disks. Today, accretion disks in binary systems are most widely discussed in cataclysmic variables. Our knowledge of the disk structures in these binaries has been recently reviewed by Horne (1991), therefore only certain points will be discussed here.

In CVs the dominant light source is the accretion disk (due to the deep potential well of the mass gaining white dwarf), with significant contributions from one or more of the following: the stream/disk impact shock; the stream overflow of the disk; the stream itself. These processes, either collectively or individually, produce what is known as the "s-wave" emission. The name comes from its appearance in trailed spectrograms (Kaitchuck, Schlegel and Hantzios 1990). Figure 2 shows a trailed spectrogram of U Gem. This image was produced from over 200 spectra which were averaged into phase bins and displayed as a gray-scale image. Orbital phase increases down the image and half of the data is repeated to make 1.5 orbital cycles. See Honeycutt, Kaitchuck and Schlegel (1987) for more details and examples.

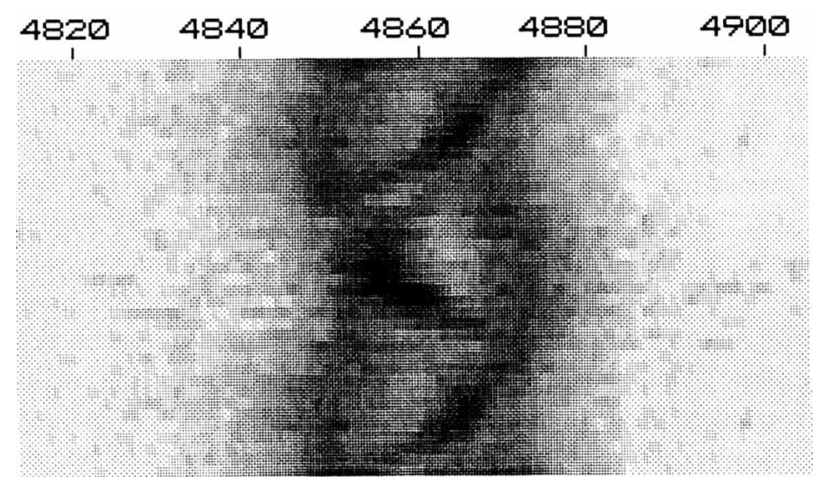

Figure 2. A trailed spectrogram of U Gem based on over 200 digital spectra. Time runs down the image with half a cycle of data repeated to show 1.5 orbital periods. The s-wave shows a complex pattern as it moves between the two Doppler disk components. 
Of course, these extra emission sources complicate our study of the disks themselves. It must also be kept in mind that the majority of the gas in these disks is optically thick in the continuum. In many cases, the emission line studies represent a minor component of the disk. In fact, it is still not entirely clear where the line emission originates. It may come from the outer edge or, more likely, from a corona-like region above and below the plane of the disk. In systems with high mass transfer rates, such as nova-like binaries and dwarf novae in outburst, the emission lines are weak or absent and instead we see broad absorption lines from the disk. In systems with low mass transfer rates, such as dwarf novae in quiescence, the emission lines are quite strong relative to the continuum. These considerations indicate that line emission may not always be the best way to learn about the bulk of the disk structure.

While continuum light may not tell us anything about the disk kinematics it can reveal details of its temperature structure. Horne (1985) has developed a technique that uses multicolor photometry of the eclipses of high inclination CVs to map the disk temperature as a function of radius. Of course, the observational data is a 1-dimensional mapping of a 2dimensional emitting surface, so there is no unique solution. Since the disk is expected (hoped) to be axisymmetric, he picks the most axisymmetric solution that fits the data. The results for RW Tri (Horne and Stiening 1985) showed a the disk to be an optically-thick radiator with colors intermediate between blackbodies and stellar atmospheres. The derived temperature-radius profile was compared to a steady-state disk model to derive a mass transfer rate of $10^{-8} \mathrm{M}_{\odot} / \mathrm{yr}$.

\section{Where Are the Line Emission Regions Located?}

While the line emission in CVs may not tell us about the bulk of the matter in the disk, it is our only source of information about the kinematics. But one of the great difficulties of studying the emission lines in CVs is the complexities of the line profiles created by the presence of the s-wave and perhaps other sources of line emission. To appreciate the complexity and diversity of the line profile behavior, one should examine the atlas of timeresolved spectroscopy of CVs by Honeycutt, Kaitchuck and Schlegel (1987).

Recently, Marsh and Horne (1988) have introduced the technique of Doppler Tomography which can be used to disentangle the emission components. While they have used a maximum entropy algorithm to process the data, almost as much can be learned by a much simpler technique of "back projection" (Horne 1991; Kaitchuck 1991). In this technique, the line profile observed at any phase is pictured as a projection of a velocity space image along the line of sight at that phase angle. To build a velocity image, a coordinate system is fixed in the rotating frame of the stars. The $X$ axis runs between the stars with the intersection of the $Y$ axis is at the center of mass of the system. The positive $Y$ axis points in the direction of the secondary star's motion. The $\mathrm{Z}$ axis is then perpendicular to the orbital plane. This coordinate system rotates with the stars. Any point on this coordinate grid will have a particular velocity vector $\left(\mathrm{V}_{\mathrm{x}}, \mathrm{V}_{\mathrm{y}}\right)$ associated with it. If it were a site of line emission, it would trace a sinusoidal curve in a trailed spectrogram. But, in the $\mathrm{V}_{\mathbf{x}}-\mathrm{V}_{\mathbf{y}}$ plane it would correspond to a point. A trailed spectrogram is a collection of sinusoids produced by each emitting point 
in the binary rest frame. To convert the trailed spectrogram into a velocity space map, the $V_{x^{-}}$ $\mathrm{V}_{\mathrm{y}}$ plane is pictured as being composed of a series of "pixels" in much the same way as a CCD image. For each pixel (each $\mathrm{V}_{\mathbf{x}}, \mathrm{V}_{\mathbf{y}}$ pair) the observed radial velocity is calculated by

$$
\mathrm{V}(\phi)=\mathrm{V}_{\mathrm{x}} \cos (\phi)+\mathrm{V}_{\mathrm{y}} \sin (\phi)
$$

where $\phi$ is the orbital phase. Spectra from all orbital phases are averaged into phase bins (typically 20-30). In each phase bin, the line flux at the calculated radial velocity is summed. This then represents the flux at this point in velocity space. The result is a velocity space map which can be viewed as a gray-scale or false-color image.

The velocity maps are a little difficult to understand at first. The double-peaked emission line profiles from a symmetric disk become a donut-shaped region in this map. The disk is turned inside-out because the high velocity gas of the inner disk (extreme line wings) falls at the outer portions of the donut, and the low velocity gas of the outer disk maps to near the origin. The advantage of viewing the data in velocity space is that many details are easier to recognize. For instance, emission from a localized region of the disk, which in a trailed spectrogram appears as a broad sinusoid which is out of phase with the orbital motion, appears as a localized spot in the velocity map. The phasing in the trailed spectrogram now translates into a location in the map which can often be interpreted as a physical location in the stellar rest frame. As another example, if the emission were coming from along the length of the stream, this would produce a pattern in the trailed spectrogram which would not be easily recognized. But in the velocity map, the stream trajectory has a very predictable location and is easily interpreted.

Application of this technique to the very complex line profile structure of $U$ Gem seen in Figure 2 results in the velocity-space map of Figure 3. The donut-shaped feature of the disk is clearly seen as is an elongated emission region which extends to the left from the expected location of the secondary star on the y-axis. These data have been analyzed by Marsh et al. (1990). They found that this emission region did not fall on the expected stream trajectory but instead appeared to arise on the secondary star and from the portion of the stream that is slowed after it passes the stream/disk shock region.

Application of this technique to several CVs (Kaitchuck et al., in preparation) has shown some fascinating features and differences between systems. Binaries like DQ Her and IP Peg show very clumpy emission in the donut region indicating very clumpy azimuthal emission structure in the disks. Most nova-like systems show little or no indication of disk line emission but instead are dominated by emission from the s-wave region or some other source.

\section{Future Directions}

Doppler mapping is a new and important tool in our detecting and understanding of disk emission. The application of Doppler emission line mapping of CVs has just begun and it will undoubtedly reveal new aspects of the disk structure. Equally exciting is the possible application of this technique to Algol-type binaries. The underlying stellar absorption profiles 
will not adversely affect Doppler mapping the way they do most other techniques. Full orbit studies of long-period Algols may give us our best look at disks which do not have the complications of CVs or the short-period Algols. They may be the disks which we can most directly compare with theory.

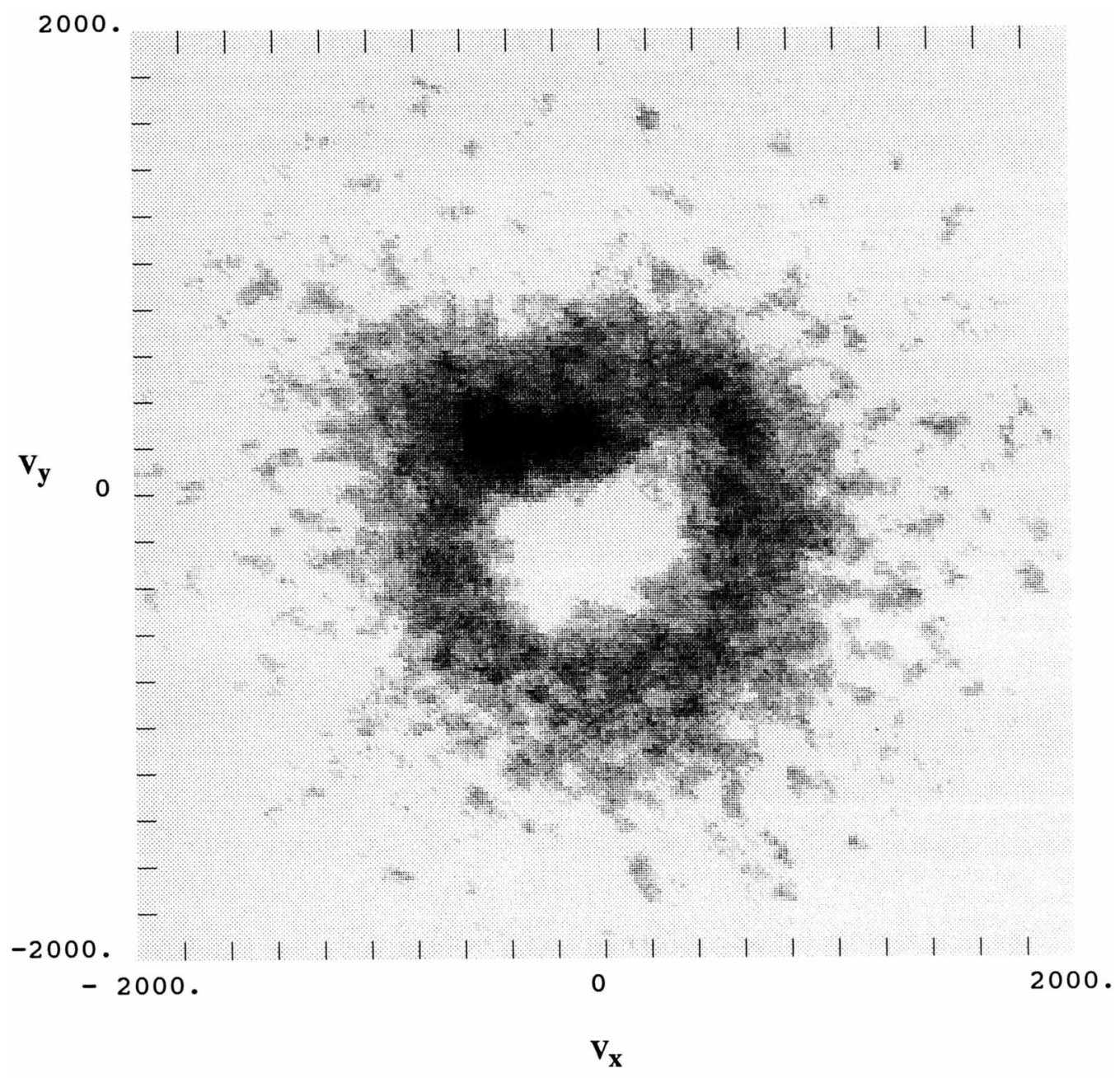

Figure 3. The velocity space map of the data in Figure 2. In this gray-scale representation the brightest areas are represented in black. The donut shaped region is due to the double-peaked disk profile. The swave emitting region is the intense elongated region running horizontally in the figure. 


\section{References}

Budding, E. 1980, in Close Binary Stars: Observations and Interpretations, I. A. U. Symposium No. 88, M. J. Plavec, D. M. Popper, and R. K. Ulrich, eds. (Dordrecht: Reidel), p. 229.

Crawford, J. A. 1955, Ap. J., 121, 71.

Crawford, R. C. 1981, Ph.D. Dissertation, University of California, Los Angeles.

Frank, J., King, A. R., and Raine, D. J. 1985, Accretion Power in Astrophysics, (Cambridge: Cambridge).

Honeycutt, R. K., Kaitchuck R. H., and Schlegel, E. M. 1987, Ap. J. Sup.,65, 451.

Honeycutt, R. K., Vesper, D. N., Yoon, T. S., and White, J. C. 1991, Proceedings of the November 1990 Pacific Rim Colloquium on New Frontiers in Binary Star Research, I. S. Nha and K. C. Leung eds., in press.

Horne, K. 1985, M. N. R. A. S., 213, 129.

Horne, K., and Stien, R. F. 1985, M. N. R. A. S.,216, 933.

Horne, K. 1991, in Structure and Emission Properties of Accretion Disks, I. A. U. Colloquium No. 129, in press.

Horne, K. 1991, paper presented at the Twelfth North American Conference on Cataclysmic Variables and Related Objects.

Joy, A. H. 1942, Pub.A.S.P. 54, 35.

Kaitchuck, R. H., and Honeycutt, R. K. 1982 Ap.J.,258, 224.

Kaitchuck, R. H., Honeycutt, R. K., and Schlegel, E. M. 1985 Pub.A.S.P. 97, 1178.

Kaitchuck, R. H. 1988, Pub.A.S.P. 100, 594.

Kaitchuck, R. H., and Park, E. A. 1988, Ap.J. 325, 225.

Kaitchuck, R. H. 1989, Space Science Rev. 50, 51.

Kaitchuck, R. H., Honeycutt, R. K., and Faulkner, D. R. 1989, Ap.J., 339, 420.

Kaitchuck, R. H., Schlegel, E. M., and Hantzios, P. A. 1990, in Accretion-Powered Compact Binaries, C. W. Mauche, ed. (Cambridge: Cambridge), p. 83.

Kaitchuck, R. H. 1991, paper presented at the Twelfth North American Conference on Cataclysmic Variables and Related Objects.

Lubow, S. H., and Shu, F. H. 1975, Ap. J. 198, 383.

Marsh, T. R., and Horne, K. 1988, M. N. R. A. S., 235, 269.

Marsh, T. R., Horne, K., Schlegel, E. M., Honeycutt, R. K., and Kaitchuck, R. H. 1990, Ap. J., 364, 637.

Olson, E. C. 1980, Ap. J. 241, 257.

Peters, G. J. 1989, Space Science Rev. 50, 9.

Peters, G. J., and Polidan, R. S. 1984, Ap. J. 282, 745.

Plavec, M. J., and Dobias, J. J. 1983, Ap. J. 272, 206.

Richards, M. T. 1991, Ap. J., in press.

Skilling, W. T., and Richardson, R. S. 1947, Astronomy (2nd ed.; Boston: Henry Holt \& Co.).

Struve, O. 1948, Pub.A.S.P. 60, 160. . 1949, M.N.R.A.S. 109, 487.

Struve, O., and Huang, S.-S. 1957, O.N.R.A.S., 3, 161 (No. 19).

Wyse, A. B. 1934, Lick Obs. Bull. No. 464, 17, 37. 